\title{
30 \\ Sounds Good: \\ Using Digital Audio for Evaluation Feedback
}

\author{
Bob Rotheram \\ Leeds Metropolitan University
}

Feedback on student work is problematic for faculty and students in British higher education. Evaluation feedback takes faculty much time to produce and students are often dissatisfied with its quantity, timing, and clarity. The Sounds Good project has been experimenting with the use of digital audio for feedback, aiming to save faculty time and give students richer learning experiences. Results are generally positive, with students and faculty acknowledging the higher quality of audio feedback. Favourable circumstances for timesaving have been identified and there is reason to believe that more faculty will eventually be able to save time on feedback without loss of quality. Practice recommendations for the use of audio feedback are given. Some issues for further research are identified.

\section{Introduction}

Ceedback on student work is a problem in UK
higher education. On the one hand, students often complain that they get too little too late, and sometimes struggle to read or understand what they do get. On the other hand, faculty may be heard grumbling that they spend ages evaluating assignments, but students are only interested in their grade, so don't read the feedback. Worse, it's said, some don't even bother to collect it! Could technology help? Years ago, Rust (2001) suggested using audiotape:

While reducing the time you spend, this may actually increase rather than reduce the amount of feedback given ... Students frequently say that they get far more information from taped comments, including the tone of one's voice, than they do from written comments, and they also do not have to try to cope with some of our illegible writing. (p. 22)

But the idea didn't catch on in the UK. Perhaps it was partly because of the clunky technology of the time: audio cassettes. Since then, digital audio has arrived: 'ripping' music CDs, Skype, and the 'Listen Again' facility on the BBC website are but a few 
examples; many students have MP3 players. Digital audio is easy to record, manipulate, and transport. I wondered whether it would be worth trying to use it for feedback.

My first experience (Rotheram, 2007) was very positive and encouraging. The students were new faculty on a postgraduate programme aiming to equip them as teachers in higher education. On the programme, the norm was for evaluators to provide extensive feedback on each assignment - 500 words or so. This used to take me quite a while to write, but I was soon able to save time by recording my comments with the free software package 'Audacity,' and sending each student an MP3 file containing their feedback. They loved it, noting its highly personal nature and the way it engaged them more than written comments. Clearly, using digital audio feedback benefited me and the students.

\section{The Sounds Good Project}

The opportunity for a larger trial came when the Users and Innovation programme of the UK's Joint Information Systems Committee (JISC) funded Sounds Good: Quicker, Better Assessment Using Audio Feedback. Running between January and July 2008, this pilot project involved 17 faculty members at Leeds Metropolitan University (Leeds Met), who were teaching various subjects at different educational levels. They used digital audio (video, in one instance) to record formative and summative feedback on students' coursework, mainly in MP3 format. The feedback was delivered through several channels, including email and a virtual learning environment. Student numbers on the various modules ranged from six to 151 . At least 463 students received one or more items of audio or video feedback.

We can't rely heavily on the results. The team were probably atypical higher education teachers: volunteers, with most having been recognised by the University for their abilities in, and commitment to, learning and teaching. Also, their project activities differed considerably. In truth, Sounds Good generated 16 case studies, some very small, about the use of digital audio or video.

That said, what learning points have emerged? The project plan contained five main evaluation questions to be explored.

\section{1. (Without reducing the amount of feedback) in what circumstances can using digital audio save evaluators' time?}

Only a small minority of faculty said that using digital audio saved them time; the largest group thought it took about the same time; the rest felt digital audio or video feedback took more time than their usual methods.

This might be regarded as disappointing. However, most team members expressed satisfaction and noted that students received more, higher-quality, feedback than they otherwise would have done. Some acknowledged that they became quicker as they grew familiar with the technology. Those who found audio feedback took them more time, or about the same time, only used it with a small number of students. So, arguably, they had not achieved full familiarity by the end of their involvement.

An evaluator seems most likely to save time by using audio when s/he: gives a substantial amount of feedback; is comfortable with the technology; writes slowly but records speech quickly.

But let's not rush to judgement. Two team members suggested it may be worth looking beyond the simple, short-term, matter of whether using audio feedback can save faculty time. One noted that he and his colleagues saved time overall by using audio to give extra advice to students on a piece of work as well as to provide feedback. The audio guidance reduced the need to clarify in other ways what was required with an assignment. Another, highly-experienced member of the team took the view that:

Giving students richer feedback will save you time anyway, as they take more notice of the feedback, and need less repeated feedback, and need less critical feedback in future anyway as their work is better, so it saves you time. 


\section{Does digital audio feedback improve students' learning experience?}

Students were overwhelmingly positive about receiving audio feedback on their coursework. They approved of its personal nature and detail - evidence that the teacher had carefully considered their work. Some appreciated the advantage of replaying the recording. Others noted that audio made it easier to grasp what the teacher felt was most important, or that it helped them to better understand why they had received a particular mark. Students whose first language is not English were pleased that it gave additional practice with their listening skills. A student with dyslexia said it was easier to listen than to read.

On the other hand, some students had reservations. A minority said they preferred written feedback; a few asked for audio and written comments on their work. Why? Some noted that it was quicker to skim-read a piece of text than to listen to an audio recording to find the passage of particular interest. But how seriously should we take this reservation? Many teachers complain that students pay little attention to written feedback. Perhaps it is an advantage that students find it harder to skim audio feedback!

\section{What do evaluators think of digital audio as a medium for providing feedback to students?}

The Sounds Good team was, on balance, strongly in favour of audio feedback. Some noted it was more likely than written comments to include examples or show how the work might be improved. A language tutor said, "it's an interesting and personal way to do the feedback for language students." Some remarked positively that they used more natural language when speaking, rather than writing, their feedback. This, one teacher thought, made it more understandable to students, particularly when it contained 'feedforward' implications for future assignments. One team member noted that students found her voice and tone reassuring and comforting. Another, citing widening participation initiatives, reported that audio feedback "is an ideal medium to assist in the development of skills and confidence."

In only one instance, a teacher discontinued use of audio feedback, and she had good reason. This evaluator thought it appropriate for a particular student assignment only to give brief feedback, and she was, in any case, a quick typist and writer. So, providing feedback via audio introduced an additional time 'overhead' for each of the 80 students in the course. This case illustrates what may be the least promising circumstances for using audio feedback.

\section{What recommendations are there for improved practice?}

The Sounds Good report (Rotheram, 2008) contains more detailed recommendations than space permits here. Briefly, experience suggests that evaluators should not expect to save time immediately by giving audio rather than written feedback. As with most new skills, it takes a while to become competent, longer to become expert. Some persistence will be required. It may feel fairly comfortable after 10-20 attempts.

How much time instructors eventually save will depend on factors such as how much feedback they give and how quickly they write. After some practice, audio may allow noticeably more feedback to be given without spending much longer on the task.

A handheld audio recorder will probably be more convenient than using a microphone connected to a computer. A handheld recorder should: a) be able to record directly to MP3 (many can't); and b) have a USB port, for easy upload to a computer. It is advisable to aim for the minimum acceptable sound quality for the particular purpose. About four minutes per megabyte (32kbps MP3) will probably be good enough for individual feedback. It is also important to ensure key administrative and qualityassurance personnel accept that audio, rather than written, feedback is being given.

It is usually better to keep the files short not to 'overdo it.' Often two or three minutes will be enough; there should be good reason to go beyond five minutes. One such reason might be that the assignment has failed and the student would benefit from further guidance before resubmission. Another might be that students are postgraduates who expect detailed feedback on drafts.

Keeping this in mind, below is an outline 
of my most recent practice when commenting on a typical essay or report using an MP3 recording.

I have the assignment details and assessment criteria with me. I read the assignment, making written comments. If it's on paper, I jot things in the margin. If it's in an electronic format (e.g. Word), I use the 'Track Changes' feature to annotate the document. I then re-read the assignment, more quickly this time, perhaps making a few more comments along the way. I jot down (on scrap paper) the main summary points I wish to make.

When ready, I start the MP3 recorder. I introduce myself to the student in a friendly manner, name the assignment I'm commenting on, and outline the main elements of the comments, which I'll be giving. I work steadily through the assignment, amplifying and explaining notes I've written in the margins and, especially at the end, making more general points, all while referring to the evaluation criteria. I explain my thought processes as I move towards allocating a grade. I then give the grade, offer a few (reasonably attainable) suggestions for improvement, even if the work is excellent, and invite comments from the student.

I build the feedback in chunks, making frequent use of the pause button. I don't bother to erase and re-record 'misspeaks;' instead, I just correct them immediately, as in conversation. When complete, I review the recording to be sure I appear approachable and not pompous or overbearing.

\section{What should be explored next?}

JISC has funded Sounds Good 2, to run between September 2008 and March 2009. The Sounds Good team will continue to use audio feedback. In addition, the project design calls for six of the members to induct two Leeds Met colleagues into using audio for feedback. The techniques will also be introduced to three additional higher education institutions.

Funding is limited but, if resources permit, the project will try to explore the following questions: can faculty become quicker in providing audio feedback if they persist? Does using audio to provide more extensive guidance and richer feedback lead to saving faculty time in the medium-to-long term? How may the practice guidelines be improved? Can instructors automate the transmission of feedback files to students? Will the novelty of audio - for students and faculty - wear off with repeated use?

\section{Conclusion}

The Sounds Good project has shown that many students prefer digital audio, rather than written feedback. Nearly all of the instructors using audio feedback prefer it too, because it enables them to serve learners better. Most evaluators aren't yet saving time, but perhaps this will come with experience.

\section{References}

Rotheram, B. (2007). Using an MP3 recorder to give feedback on student assignments. Educational Developments, 8.2.

Rotheram, B. (2008). Sounds good: Quicker, better assessment using audio feedback. Retrieved October 7, 2008, from http://sites.google. com/site/soundsgooduk/downloads/Sounds_ GoodEvaluation_10.pdf

Rust, C. (2001). A briefing on assessment of large groups. Retrieved September 26, 2008, from http://teaching.econ.usyd.edu.au/UoS/ library/A\%20Briefing\%20on\%20Assessment $\% 20$ of\%20Large\%20Groups.pdf

\section{Biography}

Bob Rotheram is Project Manager of Sounds Good at Leeds Metropolitan University, United Kingdom. He is a UK National Teaching Fellow and Reader in Assessment, Learning and Teaching. 